\title{
Implicitly coupled phase fraction equations for polydisperse flows
}

\author{
Robert Keser $^{1}$ | Alberto Ceschin ${ }^{2}$ | Michele Battistoni ${ }^{3}$ \\ | Hong G. Im ${ }^{2}$ | Hrvoje Jasak ${ }^{1}$
}

${ }^{1}$ University of Zagreb, Faculty of Mechanical Engineering and Naval Architecture, Ivana Lučića 5, Zagreb, Croatia

${ }^{2}$ King Abdullah University of Science and Technology, Clean Combustion Research Center, Thuwal, Saudi Arabia

${ }^{3}$ Department of Engineering, University of Perugia, Perugia 106123, Italy

\section{Correspondence}

Robert Keser, Department of Energy, Power and Environmental Engineering, University of Zagreb, Faculty of Mechanical

Engineering and Naval Architecture, Ivana Lučića 5, Zagreb, Croatia

Email: robert.keser@fsb.hr

\section{Funding information}

King Abdullah University of Science and Technology, Grant/Award Number: OSR-2017-CRG6-3409.03; Croatian Science Foundation, Grant/Award Number: DOK-01-2018
This work presents the implementation, verification and the validation of an incompressible Eulerian multi-fluid model for polydisperse flows. The proposed model uses a novel monolithic, i.e. implicitly coupled phase continuity equation for an arbitrary number of fluids, where the breakup source and sink terms are handled implicitly in the blocksystem. The implemented model is tested for an upward bubbly flow inside a large vertical pipe. The selected flow conditions exhibit both breakup and coalescence. The grid refinement study is conducted on four structured grids with varying levels of refinement. In the validation section, the numerical results are compared to the TOPFLOW experimental measurements. The last presented test examines the performance of the novel implicitly coupled phase continuity equation to the corresponding segregated formulation and the standard segregated formulation. The performance is evaluated by comparing the conservation error over the non-linear iterations. The presented model exhibits good agreement with the experimental measurements and gives stable results on various grids with different levels of refinement. Moreover, the implicit coupling reduces the conservation error during the calculation.

Abbreviations: TOPFLOW, Transient twO Phase FLOW test facility; CFD, Computational Fluid Dynamic; DNS, Direct Numerical Simulation; PBE, Population Balance Equation; MUSIG, Multiple Size Group; I.h.s, left hand side; r.h.s., right hand side; FVM, Finite Volume Method. 


\section{KEYWORDS}

Multiphase flow, Polydisperse bubbly flow, Eulerian multi-fluid model, OpenFOAM, Validation, Verification

\section{1 | INTRODUCTION}

Multiphase flow is a general term which includes a broad spectrum of different flow regimes. This paper limits the study to polydisperse flows, which cover a flow topology where at least one phase is dispersed within the continuous phase, i.e. it is present in the form of droplets, bubbles or particles which vary in their properties, e.g. size. Computational Fluid Dynamics (CFD) offers three standard methods for describing such flows: the Direct Numerical Simulation (DNS) approach, the Lagrangian and the Eulerian approach. The DNS approach [1, 2, 3] reconstructs and tracks the interface of each dispersed element in the computational domain [4], which often results in high computational requirements and is rarely applicable for industrial level CFD simulations. The Lagrangian approach $[5,6]$ describes the continuous phase in the Eulerian frame of reference. Still, the dispersed elements are grouped into parcels which are described in the Lagrangian frame of reference. This approach is often utilised for dilute flows. i.e. when the dispersed phase fraction is low [7, 8]. The Eulerian approach describes all phases in the Eulerian frame of reference, and all phases are treated as interpenetrating continua $[9,10,11]$. This approach is suitable for a wide range of different flow regimes. Due to averaging of the conservation equations [12,13] the results give only the averaged quantities, i.e. the local small-scale phenomena are lost. The multiphase flow regime largely depends on the phase fraction of the dispersed phase. For bubbly flows, the increase of the gas phase can significantly change the flow morphology (i.e. bubble flow, slug, churn, annular, etc.). Dilute bubbly flows often tend to be monodisperse, whereas the bubble phase increase introduces bubble breakup and coalescence, which results in polydispersity and bubble size distributions.

This work utilises the Eulerian approach for incompressible polydisperse bubbly flow. To model polydisperse flow with the Eulerian approach, the standard continuity and momentum equations need to be linked with the population balance equation (PBE). The most common choice is the two-fluid method [10,14], where only one bubble size (Sauter mean diameter) represents the dispersed phase. This approach is not suitable to describe the variance in velocity and spatial bubble distribution, because of the strong dependence of interfacial forces (such as lift, drag, wall-lubrication, etc.) on the bubble size. The Tomiyama lift force correlation $[15,16]$ even predicts a change in the force sign, which contributes to the demixing of larger and smaller bubbles. In order to improve the accuracy of the model for polydisperse flows, this work uses the multi-fluid formulation. This approach allows every bubble size to be treated as a different phase in the calculation.

Furthermore, to solve the PBE, several different approaches are available [17]: sectional and class methods, method of moments, quadrature-based moment methods and Monte Carlo methods. Various recent studies [18, $19,20,21,22,23,24]$ employed quadrature-based moment methods to describe polydisperse bubbly flows. All the mentioned approaches are still an active area of research. Engineers and researchers are continuously assessing which formulation is most suitable for the problem at hand. In this paper, the class method (CM) is selected, and the implementation details are discussed later in Section 2.

Due to a large number of sub-models and derivation assumptions, which are required for accurate predictive capabilities, the Eulerian multi-fluid model often exhibits stability issues. Some of the issues are a result of lacking hyperbolicity, improper closure models and unphysical values of the phase fraction variables [25, 26, 27, 24, 28, 29, 30, 31]. Furthermore, the addition of numerous phase fraction equations (with breakup and coalescence source/sink terms) to the algorithm introduces additional problems with the conservation and boundedness of the solution. Therefore, 
this paper further upgrades the implicitly coupled phase fraction formulation [32] with breakup and coalescence functionality. This implementation takes advantage of the reformulation procedure described by [30], which contributes to the boundedness of the solution at both ends. The novel implicitly coupled phase fraction solution procedure is tested for an upward bubbly flow inside a large vertical pipe which exhibits both breakup and coalescence. Moreover, the performance of the proposed implicitly coupled implementation is compared to the corresponding segregated reformulated version and the segregated standard formulation of the phase fraction equation. The implementation of the proposed model is done within foam-extend, using the available framework for the development of block-coupled solvers [33, 29, 34].

The remainder of the paper is arranged in four sections. Section 2 gives the governing equations for the chosen multi-fluid model, which is later used for prediction of polydisperse bubbly flows with an arbitrary number of fluids. Section 3 deals with the numerical model, i.e. it gives an overview of the selected solution algorithm and the numerical procedures. Furthermore, it presents details of the model implementation, and special attention is given to the breakup and coalescence model treatment for the implicitly coupled phase continuity equation formulation. Section 4 includes the grid refinement study and the validation part, which compares the numerical results to available experimental data. Additionally, it also gives a detailed performance analysis, where the implicitly coupled phase continuity equation is compared with the corresponding segregated and the standard segregated formulation. The conclusions regarding the behaviour of the implemented model are drawn in Section 5.

\section{2 | FORMULATION OF THE COMPUTATIONAL MODEL}

This section describes the Eulerian multi-fluid model, which is implemented within this work. Furthermore, the model is generalised for an arbitrary number of incompressible fluids. The presented model is an upgrade of the work given in [32], which utilises the conditionally averaged equations for the incompressible multi-fluid model (which follows the procedure described by [35], [30] and [13]). In this work, the model is enhanced with breakup and coalescence capability, and the turbulence model is updated to account for bubbly flow at higher phase fractions. Moreover, the interfacial momentum transfer models were replaced with more advanced formulations which cover a broader range of flow conditions.

As previously mentioned, to successfully model polydisperse flows using the Eulerian multi-fluid framework, the standard continuity and momentum equations need to be linked with the PBE. Furthermore, to solve the PBE, the classes method is used to discretise the internal coordinates, i.e. bubble diameters into a finite number of classes. This approach is utilised by the widely available Multiple Size Group (MUSIG) model [36, 21] and the Inhomogeneous (or Heterogeneous) MUSIG model [37, 38], where the PBE is practically converted into a size fraction equation (a continuity equation for the size group). In comparison with the MUSIG and I-MUSIG model, this work employs the fully inhomogeneous (or the full multiphase) approach. Here, every bubble class has its own phase continuity and phase momentum equation or using the I-MUSIG terminology each velocity group has only one bubble size class.

The bubble diameters are discretised using the equal diameter distribution, i.e. the $i$-th bubble diameter $d_{i}$ is calculated from:

$$
\begin{gathered}
d_{i}=d_{\min }+\Delta d\left(i-\frac{1}{2}\right), \\
\Delta d=\frac{d_{\text {max }}-d_{\min }}{n_{\text {bubbles }}},
\end{gathered}
$$


where $d_{\max }$ and $d_{\min }$ are the maximum and minimal bubble diameter and $n_{\text {bubbles }}$ is the total number of bubble classes.

\section{1 | Phase-intensive momentum equation}

The conditionally averaged momentum equation for phase $\varphi$ in the phase-intensive formulation is given by:

$$
\frac{\partial \overline{\mathbf{U}}_{\varphi}}{\partial t}+\overline{\mathbf{U}}_{\varphi} \nabla \cdot \overline{\mathbf{U}}_{\varphi}+\nabla \cdot \overline{\mathbf{R}}_{\varphi}^{\mathrm{eff}}+\frac{\nabla \alpha_{\varphi}}{\alpha_{\varphi}} \cdot \overline{\mathbf{R}}_{\varphi}^{\text {eff }}=-\frac{\nabla \bar{p}}{\bar{\rho}_{\varphi}}+\mathbf{g}+\frac{\overline{\mathbf{M}}_{\varphi}}{\alpha_{\varphi} \bar{\rho}_{\varphi}}+\frac{\mathbf{S}_{\mathrm{M} \varphi}}{\alpha_{\varphi} \bar{\rho}_{\varphi}}
$$

where $\overline{\mathbf{U}}_{\varphi}$ indicates the averaged phase velocity, $\alpha_{\varphi}$ is the phase fraction, $\overline{\mathbf{R}}_{\varphi}^{\text {eff }}$ is the joined viscous and turbulent stress, $\bar{p}$ is the mixture pressure, $\bar{\rho}_{\varphi}$ is the phase density, $\mathbf{g}$ is the gravitational acceleration, $\overline{\mathbf{M}}_{\varphi}$ is the averaged interfacial momentum transfer term, and $\mathbf{S}_{\mathrm{M} \varphi}$ is the net momentum source term due to breakup and coalescence processes (caused by the transfer of mass between the bubble classes). A detailed description and implementation of individual terms are given in [32, 35, 30, 13]. To avoid singularities in eq. (3) when $\alpha_{\varphi}$ approaches zero, source terms which contain $\alpha_{\varphi}$ in the denominator need to be implemented carefully. The $\alpha_{\varphi}$ term in the denominator is implemented as $\max \left(\alpha_{\varphi}, \delta\right)$, where $\delta$ is a very small number (e.g. $\left.10^{-20}\right)$. In most cases, the $\alpha_{\varphi}$ term in the denominator gets cancelled out with the $\alpha_{\varphi}$ term in the numerator originating from the interfacial transfer model, which shall be given later in the text.

In its general form, the interfacial momentum transfer term $\overline{\mathbf{M}}_{\varphi}$ reads:

$$
\overline{\mathbf{M}}_{\varphi}=\sum_{i=1, i \neq \varphi}^{n_{\text {phases }}} \overline{\mathbf{M}}_{\varphi, i}
$$

where $n_{\text {phases }}$ is the total number of fluids, and $\overline{\mathbf{M}}_{\varphi, i}=-\overline{\mathbf{M}}_{i, \varphi}$ denotes the momentum transferred between phases $\varphi$ and $i$.

The presented work limits the model to bubbly flows, where the water is described by only one continuous phase, and the air phase is split into an arbitrary number of classes depending on the bubble diameter. Therefore, $n_{\text {phases }}=n_{\text {bubbles }}+1$, where $n_{\text {bubbles }}$ is the total number of bubble classes. The bubble phases exchange momentum with the continuous phase, i.e. water via wall-lubrication, virtual mass, lift, drag and turbulent dispersion force:

$$
\begin{array}{rll}
\overline{\mathbf{M}}_{\mathrm{d}, i}= & \alpha_{\mathrm{d}, i} C_{\mathrm{d}, i} \frac{3}{4} \frac{\bar{\rho}_{\mathrm{c}}}{d_{i}}\left|\overline{\mathbf{U}}_{\mathrm{r}, i}\right| \overline{\mathbf{U}}_{\mathrm{r}, i} \quad \text { drag } \\
& +\alpha_{\mathrm{d}, i} C_{\mathrm{l}, i} \bar{\rho}_{\mathrm{c}} \overline{\mathbf{U}}_{\mathrm{r}, i} \times\left(\nabla \times \overline{\mathbf{U}}_{\mathrm{c}}\right) \quad \text { lift } \\
& +\alpha_{\mathrm{d}, i} C_{\mathrm{vm}} \bar{\rho}_{\mathrm{c}}\left(\frac{D_{\mathrm{c}} \overline{\mathbf{U}}_{\mathrm{c}}}{D t}-\frac{D_{\mathrm{d}, i} \overline{\mathbf{U}}_{\mathrm{d}, i}}{D t}\right) \quad \text { virtual mass } \\
& +C_{\mathrm{d}, i} \frac{3}{4} \alpha_{\mathrm{d}, i} \frac{\bar{\rho}_{\mathrm{c}}}{d_{i}} \frac{v_{\mathrm{c}}^{\mathrm{t}}}{\sigma_{\alpha}}\left|\overline{\mathbf{U}}_{\mathrm{r}, i}\right|\left(\frac{\nabla \alpha_{\mathrm{c}}}{\alpha_{\mathrm{c}}}-\frac{\nabla \alpha_{\mathrm{d}, i}}{\alpha_{\mathrm{d}, i}}\right) \text { turbulent dispersion } \\
& +C_{\mathrm{wl}, i} \alpha_{\mathrm{d}, i} \bar{\rho}_{\mathrm{c}}\left|\overline{\mathbf{U}}_{\mathrm{r}, i}\right|^{2} \mathrm{n}_{\mathrm{W}} \quad \text { wall lubrication force. }
\end{array}
$$

where the subscript d signifies the dispersed phase, and the subscript c denotes the continuous phase. The $D_{\varphi} \overline{\mathbf{U}}_{\varphi} / D t$ term indicates the phase material time derivative. The relative velocity term is calculated as $\overline{\mathbf{U}}_{\mathrm{r}, i}=\overline{\mathbf{U}}_{\mathrm{c}}-\overline{\mathbf{U}}_{\mathrm{d}, i} \cdot C_{\mathrm{d}, i}, C_{\mathrm{l}, i}$, $C_{\mathrm{wl}, i}$ are the drag, lift and the wall lubrication coefficient of the $i$-th bubble phase. The diameter of the $i$-th dispersed 
phase is given with $d_{i}$. In this work, the virtual mass coefficient $C_{\mathrm{vm}}$ is treated as a constant, which has the same value for all bubble classes $C_{\mathrm{vm}}=0.5$.

The drag coefficient of every bubble class $C_{\mathrm{d}, i}$ is evaluated using the GRACE drag model [39]. The bubble class lift force coefficient $C_{\mathrm{l}, i}$ is calculated using the Tomiyama lift model $[15,16]$. The wall lubrication force coefficient $C_{\mathrm{wl}, i}$ is estimated with the Tomiyama wall lubrication model [40], and the turbulent dispersion force is determined using the Favre averaged drag (FAD) model [41].

The interfacial momentum transfer term for the water phase (i.e. the continuous phase) is calculated as:

$$
\overline{\mathbf{M}}_{\mathrm{c}}=-\sum_{i=1}^{n_{\text {bubbles }}} \overline{\mathbf{M}}_{\mathrm{d}, i}
$$

\section{2 | Phase continuity equation}

For incompressible flows, the standard phase continuity equation formulation (for phase $\varphi$ ) is given by:

$$
\frac{\partial \alpha_{\varphi}}{\partial t}+\nabla_{\bullet}\left(\overline{\mathbf{U}}_{\varphi} \alpha_{\varphi}\right)=\frac{S_{\varphi}}{\rho_{\varphi}}
$$

where $S_{\varphi}$ is the net source term due to mass transfer between bubble classes caused by breakup and coalescence, which is going to be described in Section 2.3.

Following the original procedure described by [30], which was re-derived for the multi-fluid formulation by [42, 43], the modified phase continuity equation reads:

$$
\frac{\partial \alpha_{i}}{\partial t}+\nabla \cdot\left(\overline{\mathbf{U}} \alpha_{i}\right)+\nabla \cdot\left(\alpha_{i} \sum_{j=1, j \neq i}^{n_{\text {phases }}} \alpha_{j}\left(\overline{\mathbf{U}}_{i}-\overline{\mathbf{U}}_{j}\right)\right)=\frac{s_{i}}{\rho_{i}}
$$

The modified formulation employs the mixture velocity $\bar{U}$, which is the only velocity guaranteed to be divergencefree (when solving for the mixture pressure). Furthermore, the third term on the I.h.s. introduces direct cross-coupling of the phase continuity equations. These properties contribute to the boundedness of the solution at both ends. Due to the non-linearity of the cross-coupling term, the equations were linearised and implemented in the block-system for an arbitrary number of fluids [32]. In this work, the implicitly-coupled solution procedure is further improved by adding the breakup and coalescence functionality, which contributes to stronger cross-coupling of the phase continuity equations. The selected breakup and coalescence models are described and presented in Section 2.3. The boundedness of the proposed model is further investigated in Section 3.2.

\section{3 | Breakup and coalescence}

This section describes the calculation of the net source term $S_{\varphi}$ in eq. (7), which represents the transfer of mass between phases due to breakup and coalescence. The continuous phase, i.e. the water phase, does not undergo breakup nor coalescence. Therefore, $S_{\mathrm{c}}=0$. On the other hand, the air phase, and the corresponding bubble classes undergo breakup and coalescence, and the net source term for the $i$-th bubble class can be divided in the following 
manner:

$$
S_{\mathrm{d}, i}=B_{\mathrm{B}, \mathrm{d}, i}-D_{\mathrm{B}, \mathrm{d}, i}+B_{\mathrm{C}, \mathrm{d}, i}-D_{\mathrm{C}, \mathrm{d}, i}
$$

where $B_{\mathrm{B}, \mathrm{d}, i}$ is the bubble birth rate due to breakup from larger bubbles (into class $i$ ), $D_{\mathrm{B}, \mathrm{d}, i}$ is the bubble death rate due to breakup (from class $i$ ) into smaller bubbles. Similarly, $B_{\mathrm{C}, \mathrm{d}, i}$ is the bubble birth rate due to coalescence of smaller bubble classes (into class $i$ ), and $D_{\mathrm{C}, \mathrm{d}, i}$ is the bubble death rate due to the coalescence of smaller bubble classes (including class $i$ ) into larger ones.

To satisfy the conservation requirements, the formulation of the breakup and coalescence model need to guarantee that the total source is zero when summed over all bubble classes:

$$
\sum_{i}^{n_{\text {bubbles }}}\left(B_{\mathrm{B}, \mathrm{d}, i}-D_{\mathrm{B}, \mathrm{d}, i}\right)=0 \text {, }
$$

and

$$
\sum_{i}^{n_{\text {bubbles }}}\left(B_{\mathrm{C}, \mathrm{d}, i}-D_{\mathrm{C}, \mathrm{d}, i}\right)=0
$$

In this work, the bubble breakup is modelled according to [44], and coalescence modelling follows the procedure described by [45]. The corresponding source/sink terms are given by:

$$
\begin{aligned}
& B_{\mathrm{B}, \mathrm{d}, i}=\sum_{j>i}^{n_{\text {bubbles }}} B_{j, i} \rho_{\mathrm{d}, j} \alpha_{\mathrm{d}, j} \\
& D_{\mathrm{B}, \mathrm{d}, i}=\rho_{\mathrm{d}, i} \alpha_{\mathrm{d}, i} \sum_{j<i}^{n_{\text {bubbles }}} B_{i, j} \\
& B_{\mathrm{C}, \mathrm{d}, i}=\frac{1}{2} \sum_{j \leq i}^{n_{\text {bubbles }}} \sum_{k \leq i}^{n_{\text {bubbles }}} C_{j, k} X_{j, k, i} \rho_{\mathrm{d}, j} \alpha_{\mathrm{d}, j} \rho_{\mathrm{d}, k} \alpha_{\mathrm{d}, k} \frac{m_{j}+m_{k}}{m_{j} m_{k}}, \\
& D_{\mathrm{C}, \mathrm{d}, i}=\sum_{j}^{n_{\text {bubbles }}} C_{i, j} \rho_{\mathrm{d}, i} \alpha_{\mathrm{d}, i} \rho_{\mathrm{d}, j} \alpha_{\mathrm{d}, j} \frac{1}{m_{j}}
\end{aligned}
$$

where $B_{i, j}$ and $C_{i, j}$ are bubble breakup and coalescence rates of class $i$ with $j, m$ is the representative bubble mass of a particular class, and $X_{j, k, i}$ is the coalescence mass matrix which defines the fraction of mass transfered from classes $j$ and $k$ to class $i$.

Model details and implementation guidelines for the mentioned breakup and coalescence mechanisms are given in $[44,45,39]$.

As suggested by [44], the integrand in the definition of the breakup rate is implemented using incomplete gamma functions to decrease the computational load during the calculation. Furthermore, it is beneficial to point out that 
several implementations of the Luo and Svendsen model $[39,37]$ introduce a calibration coefficient $F_{\mathrm{B}}$ which is used for the fine-tuning of the model.

\section{4 | Turbulence model}

This work employs the mixture $k-\epsilon$ turbulence model presented by [46], where the derivation of the mixture transport equation for the turbulence quantities is based on the summation of the corresponding phase-averaged transport equations. Following the original notation the mixture equations read:

$$
\frac{\partial\left(\rho_{\mathrm{m}} k_{\mathrm{m}}\right)}{\partial t}+\nabla_{\bullet}\left(\rho_{\mathrm{m}} \widetilde{\mathbf{U}}_{\mathrm{m}} k_{\mathrm{m}}\right)=\nabla_{\bullet} \frac{\mu_{\mathrm{m}}^{\mathrm{t}}}{\sigma_{\mathrm{m}}} \nabla k_{\mathrm{m}}+P_{k}^{\mathrm{m}}-\rho_{\mathrm{m}} \epsilon_{\mathrm{m}}+S_{k}^{\mathrm{m}},
$$

and

$$
\frac{\partial\left(\rho_{\mathrm{m}} \epsilon_{\mathrm{m}}\right)}{\partial t}+\nabla_{\bullet} \cdot\left(\rho_{\mathrm{m}} \widetilde{\mathbf{U}}_{\mathrm{m}} \epsilon_{\mathrm{m}}\right)=\nabla \cdot \frac{\mu_{\mathrm{m}}^{\mathrm{t}}}{\sigma_{\mathrm{m}}} \nabla \epsilon_{\mathrm{m}}+\frac{\epsilon_{\mathrm{m}}}{k_{\mathrm{m}}}\left(C_{\epsilon 1} P_{k}^{\mathrm{m}}-C_{\epsilon 2} \rho_{\mathrm{m}} \epsilon_{\mathrm{m}}\right)+C_{\epsilon 3} \frac{\epsilon_{\mathrm{m}}}{k_{\mathrm{m}}} S_{k}^{\mathrm{m}}
$$

where the subscript $\mathrm{m}$ denotes the mixture. Therefore, $\rho_{\mathrm{m}}, \mu_{\mathrm{m}}^{\mathrm{t}}, k_{\mathrm{m}}$ and $\epsilon_{\mathrm{m}}$ denote the density, turbulent dynamic viscosity, turbulence kinetic energy and the dissipation of turbulence energy of the mixture. It should be emphasized that the mixture properties are mass-weighted to remove additional terms which contain the spurious derivatives. $\sigma_{\mathrm{m}}$, $C_{\epsilon 1}, C_{\epsilon 2}$ and $C_{\epsilon 3}$ are modifiable model constants. Finally, the $S_{k}^{\mathrm{m}}$ source term pertains to inter-phase energy transfer resulting from the inter-phase forces which is derived from the ensemble averaging.

A detailed description of the model and individual terms is available at [46].

\section{3 | NUMERICAL MODEL}

The proposed model is implemented in foam-extend. Therefore, the multi-fluid equations are discretised using the collocated cell-centred Finite Volume Method (FVM) [47, 48]. The solution procedure employs a combination of SIMPLE [49] and PISO [50] algorithm, where multiple PISO correctors can be utilised within every SIMPLE correction (outer-corrector) step. The utilised solution algorithm per each time step is given in Algorithm 1, where $N_{\text {SIMPLE }}$ gives

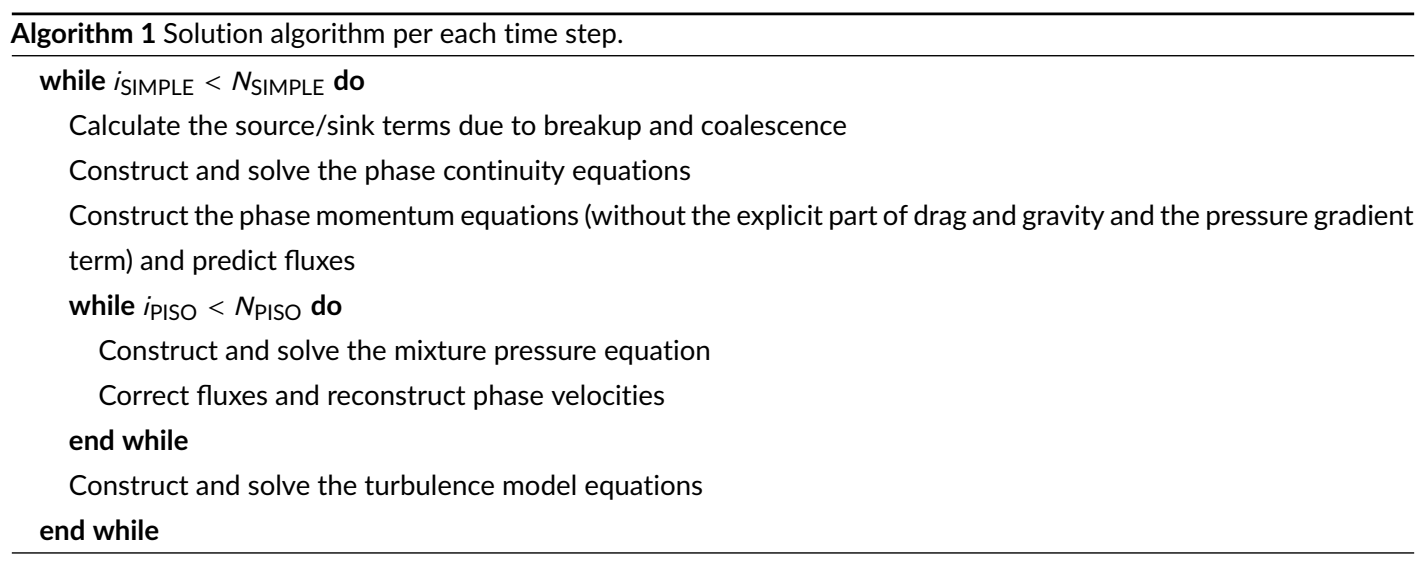




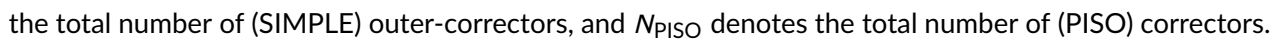

The next sub-sections give a brief overview of the block-matrix structure, accompanied by the description of three different phase continuity implementations, and a summary of the chosen linear solver settings and discretisation schemes.

\section{1 | Block-system structure}

Two levels can represent the block-system structure [33]. The first level describes the coupling due to the spatial discretisation of the domain (by applying collocated cell-centred FVM), and the second one gives the coupling among the variables. The result of the spatial discretisation using the FVM is a linear system:

$$
\left(\begin{array}{cccc}
a_{1,1} & a_{1,2} & \cdots & a_{1, N} \\
a_{2,1} & a_{2,2} & \cdots & a_{2, N} \\
\vdots & \vdots & \ddots & \vdots \\
a_{N, 1} & a_{N, 2} & \cdots & a_{N, N}
\end{array}\right)\left(\begin{array}{c}
\phi_{1} \\
\phi_{2} \\
\vdots \\
\phi_{N}
\end{array}\right)=\left(\begin{array}{c}
b_{1} \\
b_{2} \\
\vdots \\
b_{N}
\end{array}\right)
$$

where $N$ is both the number of control volumes and the number of unknowns in the linear system. Furthermore, $\phi$ denotes cell-centred field values, a represents the matrix entries, and $b$ gives the r.h.s. values.

When using the segregated approach, the matrix entries, field values and the r.h.s. values are scalars, since the equations are solved sequentially one after another. With the implicitly coupled approach, when all equations are solved together, $\phi$ and $b$ become $n$-dimensional vectors, and the matrix entry a becomes an $n \times n$ tensor (where $n$ is the number of implicitly coupled equations).

\subsection{Implementation of the phase continuity equations}

Due to the third term on the r.h.s., i.e. the $\alpha_{i} \alpha_{j}$ product, eq. (8) is non-linear. The linearisation and implementation of eq. (8) (but without the net source term $S_{i}$ ) in the block-system is presented in [32]. In the current work, the coupled formulation is further improved by adding the breakup and coalescence source terms to the block-system. The breakup and coalescence source/sink terms, eqs. (12) to (15), introduce additional communication between the bubble phase continuity equations, which contributes to a stronger cross-coupling of the equations. Individual sink/source terms need to be investigated if they are suitable for implicit treatment.

Patankar [51] recommends splitting the net source term into the always positive source and always negative sink. The negative sink term is treated implicitly because it increases the diagonal dominance of the matrix, which is conducive to convergence. The source term is treated explicitly, which enhances the boundedness and the stability of the solution. Those instructions are suitable for segregated algorithms. The work presented in [52] gives guidelines for the treatment of source/sink terms for always positive variables in the block-system, which introduce additional implicit cross-coupling terms. The cross-coupling terms lie on the off-diagonal of the matrix entry, and they need to have an opposite sign of the implicit sink, i.e. they need to be positive on the right-hand side to preserve the diagonal dominance. Therefore, only the positive cross-coupling terms are suitable for implicit treatment.

Following the presented guidelines, the breakup and source terms are evaluated on a term by term basis. As stated before in Section 2.3, the net source term in eqs. (7) and (8) is divided into two categories, source and sink due to breakup, and source and sink due to coalescence (eq. (9)).

In the phase continuity equation for bubble class $i$ (eq. (8)), the breakup source term (eq. (12)) linearly couples 
the equation with the remaining smaller bubble classes. Since the breakup rate, density and phase fraction are always positive values, and since this term is a source (positive sign in the equation), it is an ideal candidate for the implicit cross-coupling term. The breakup sink term (eq. (13)) is also linear, and it has a negative sign on the r.h.s., which makes it suitable for an implicit sink. The whole breakup model can be treated implicitly (in terms of the bubble phase fraction).

The coalescence model implementation (eqs. (14) and (15)) has a non-linear phase fraction product, both in the source and sink term. Even the linearisation produces several terms which are not suitable for the implictly coupled implementation, e.g. negative explicit terms and negative cross-coupling terms. Furthermore, the parallel usage of explicit and implicit, i.e. old and new values of the bubble phase fraction (with the same coalescence rate), while evaluating the source and sink terms can breach the conservation criterion stated in Equation (11). Therefore, the coalescence terms are treated explicitly.

Since the usage of presented Eulerian multi-fluid model is narrowed down to bubbly flows, the implicitly coupled phase continuity equation ([30] formulation) for the $i$-th bubble class $\alpha_{\mathrm{d}, i}$ reads:

$$
\begin{aligned}
\frac{\partial \alpha_{\mathrm{d}, i}}{\partial t}+\nabla \cdot\left(\overline{\mathbf{U}} \alpha_{\mathrm{d}, i}^{\mathrm{n}}\right) & +\nabla_{\bullet}\left(\alpha_{\mathrm{d}, i}^{\mathrm{n}} \sum_{j=1, j \neq i}^{n_{\text {phases }}} \alpha_{j}^{\mathrm{o}}\left(\overline{\mathbf{U}}_{i}-\overline{\mathbf{U}}_{j}\right)\right) \\
& +\underbrace{\nabla \cdot\left(\alpha_{\mathrm{d}, i}^{\mathrm{o}} \sum_{j=1, j \neq i}^{n_{\text {phases }}} \alpha_{j}^{\mathrm{n}}\left(\overline{\mathbf{U}}_{i}-\overline{\mathbf{U}}_{j}\right)\right)}_{\text {implicit cross-coupling }} \\
& -\nabla_{\bullet}(\alpha_{\left.\mathrm{d}, i \sum_{j=1, j \neq i}^{\mathrm{o}} \sum_{\text {phases }}^{\mathrm{o}}\left(\overline{\mathbf{U}}_{i}-\overline{\mathbf{U}}_{j}\right)\right)} \underbrace{\sum_{j>i}^{n_{\text {bubbles }} B_{j, i} \frac{\rho_{\mathrm{d}, j}}{\rho_{\mathrm{d}, i}} \alpha_{\mathrm{d}, j}^{\mathrm{n}}}}
\end{aligned}
$$

and the implicitly coupled phase fraction equation for the continuous phase $\alpha_{\mathrm{c}}$ is given by:

$$
\alpha_{\mathrm{c}}^{\mathrm{n}}+\underbrace{\sum_{i=1}^{n_{\text {bubbles }}} \alpha_{\mathrm{d}, i}^{\mathrm{n}}}_{\text {implicit cross-coupling }}=1
$$

where superscript o denotes the old/explicit value and the superscript $\mathrm{n}$ gives the new/implicit value. The last term on the r.h.s. in eq. (19) $S_{\mathrm{C}, \mathrm{d}, i}$, gives the net source term due to coalescence, which is calculated explicitly.

Both segregated implementations which are utilised in this work, use the same phase continuity equation for the continuous phase, which is defined as:

$$
\alpha_{\mathrm{c}}^{\mathrm{n}}+\sum_{i=1}^{n_{\text {bubbles }}} \alpha_{\mathrm{d}, i}^{\mathrm{o}}=1
$$


The segregated Weller implementation is given by:

$$
\frac{\partial \alpha_{\mathrm{d}, i}}{\partial t}+\nabla_{\bullet}\left(\overline{\mathbf{U}} \alpha_{\mathrm{d}, i}^{\mathrm{n}}\right)+\nabla_{\bullet}\left(\alpha_{\mathrm{d}, i}^{\mathrm{n}} \sum_{j=1, j \neq i}^{n_{\text {phases }}} \alpha_{j}^{\mathrm{o}}\left(\overline{\mathbf{U}}_{i}-\overline{\mathbf{U}}_{j}\right)\right)=\frac{S_{\mathrm{d}, i}}{\rho_{\mathrm{d}, i}}
$$

and the standard segregated formulation is implemented as:

$$
\frac{\partial \alpha_{\mathrm{d}, i}}{\partial t}+\nabla \cdot\left(\overline{\mathbf{U}}_{\mathrm{d}, i} \alpha_{\mathrm{d}, i}^{\mathrm{n}}\right)=\frac{S_{\mathrm{d}, i}}{\rho_{\mathrm{d}, i}}
$$

where the last term on the r.h.s. in both eqs. (22) and (23) $S_{\mathrm{d}, i}$, gives the net source term due to breakup and coalescence, which is calculated explicitly.

When using the implicitly coupled formulation, all phase continuity equations (eq. (19)) for the selected number of bubble classes and the continuous phase continuity equation (eq. (20)) are put into a single block-matrix and solved together. Whereas, when using one of the segregated formulations all equations are solved sequentially one after another.

The proposed model uses an appropriate bounded scheme (described in the following section) for the solution of phase continuity equations, which contribute to the preservation of the $\alpha_{i} \geq 0$ constraints. The implementation of the phase continuity equation for the continuous phase (both the coupled and segregated formulations) enforces that the sum of all phase fractions is equal to 1. No additional recombination/blending techniques are employed in this work.

\section{3 | Numerical procedure}

In this work, all test cases (described in Section 4) employ the same discretisation and linear solver settings; any differences in solver settings are explicitly mentioned and emphasised.

The turbulence model equations $(k$ and $\epsilon$ ) and all phase continuity equation formulations were solved using a Bi-Conjugate Gradient Method preconditioned by DILU [53]. The mixture pressure equation employed the selection algebraic multigrid algorithm [54] preconditioned with the Gauss-Seidel smoother [33]. All linear solvers employed an absolute tolerance of $10^{-10}$ for the normalised residual value. Regarding the discretisation schemes, Laplacians, gradients and cell to face interpolations were calculated using the linear interpolation. The phase fractions and the momentum variables were advected using the linear upwind-biased approximation; only the phase fractions also applied a limiter for stronger bounding. The advection of turbulence model variables used the upwind scheme.

\section{4 | RESULTS}

This section presents a detailed description of the selected test case, and the results obtained during the mesh refinement study (Section 4.1), validation (Section 4.2) and performance testing (Section 4.3).

For testing of the implemented numerical models, the numerical results are compared against the TOPFLOW (Transient twO Phase FLOW test facility) experimental measurements [55]. The TOPFLOW experiment has been carried out at the Helmholtz-Zentrum Dresden-Rossendrof (HZDR) site, where water was circulated from the bottom to the top of a large vertical pipe with a height of 9 meters and with an internal diameter $D$ of 195.3 millimetres. The air was radially injected into the water stream through a variable gas injection systems, i.e. air chambers with 


\begin{tabular}{ccc}
\hline Sampling level & Injection length $(\mathrm{mm})$ & $L / D$ ratio \\
\hline A & 221 & 1.1 \\
D & 494 & 2.5 \\
I & 1552 & 7.9 \\
M & 4417 & 22.6 \\
\hline
\end{tabular}

TAB LE 1 Denotation and positions of sampling levels.

a large number of annular distributed orifices. The instantaneous bubble phase fraction information and the bubble size distribution were measured using a fixed wire-mesh sensor. The experiment covered a large number of different flow conditions, i.e. different combinations of superficial velocities for the water and bubble phase. In this work, we consider the measurement point 107 with $1 \mathrm{~mm}$ orifices. Table 1 gives the vertical positions of the employed sampling levels in the flow direction, and the denotations are kept the same as in the experiment [55].

To reduce the computational effort of the simulation, only a $45^{\circ}$ radial sector of the pipe is considered (symmetry boundary conditions are imposed at both vertical sides), and only the first half of the pipe's length is taken into account. The inlet boundary is located at $z=221 \mathrm{~mm}$ (level A), and the inlet boundary conditions for the bubble phases are initialised using the first available experimental measurements. The water phase is initialised using a fully developed single-phase pipe flow. The described geometry is given in Figure 1.

\section{1 | Mesh refinement study}

In this section, the grid refinement study is carried out by employing four structured grids with various refinement levels. The grid names and the corresponding number of cells are shown in Table 2. The axial and radial grid density of grid-4 is shown in Figure 1 . The remaining grids were gradually and uniformly coarsened to reduce the total number of cells.

\begin{tabular}{cc}
\hline Grid name & Total number of cells \\
\hline grid-1 & 1400 \\
\hline grid-2 & 2232 \\
\hline grid-3 & 5727 \\
grid-4 & 6900 \\
\hline
\end{tabular}

TAB LE 2 Computational grid details for the grid refinement study.

Figure 2 presents the comparison of numerical results, i.e. total radial bubble phase fraction $\alpha_{d}$ obtained with the four described grids for three different sampling levels (D, I and M). All three figures show good agreement and stable trends. Only the D level exhibits more substantial discrepancies in the peak values, which is caused by the difference in radial mesh density (which also affects the interpolation of the experimental values onto the inlet patch). The obtained accuracy in space is estimated using the ReFRESCO [56] application which employs the procedures given by [57]. The study is conducted for the minimal value of continuous phase fraction $\alpha_{\mathrm{c}}$ at sampling level $\mathrm{D}$. The input values for the evaluator application are given in Table 3. 


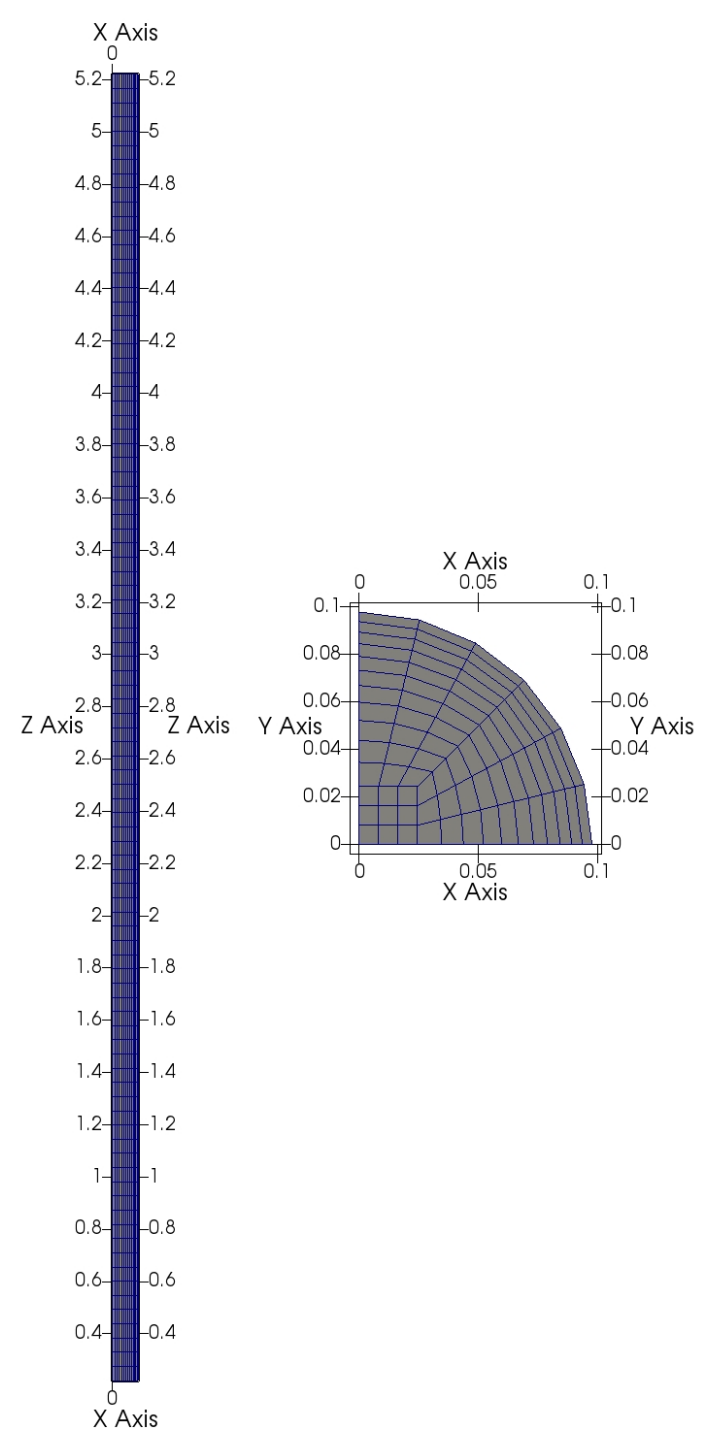

FIGURE 1 Geometry of the test case and mesh density for grid-4

The estimator output is shown in Table 4 , where $\phi_{0}$ is the extrapolated exact solution, $\phi_{1}$ is the finest level solution, $U_{\phi}$ is the uncertainty estimate, and $p$ is the achieved accuracy in space.

\section{2 | Validation}

In this section, the grid-4 numerical results from the previous section are compared with the TOPFLOW experimental measurements using the measurement point 107 (with $1 \mathrm{~mm}$ orifices). Figure 3 gives a comparison of the total radial 

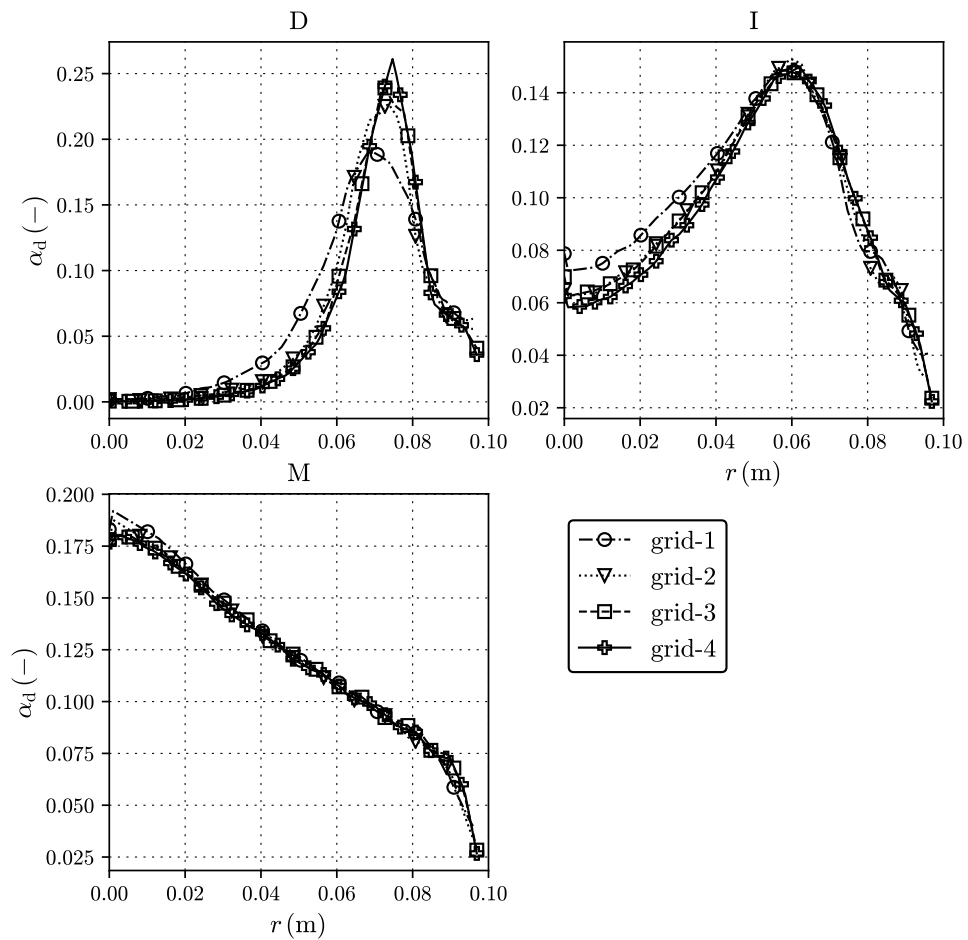

FIGURE 2 Mesh refinement study: comparison of the total radial bubble phase fraction profiles.

\begin{tabular}{cc}
\hline Grid & $\min \left(\alpha_{\mathrm{c}}\right)$ at measuring line $\mathrm{D}$ \\
\hline grid-1 & 0.8084 \\
grid-2 & 0.7731 \\
grid-3 & 0.7609 \\
grid-4 & 0.7389 \\
\hline
\end{tabular}

TABLE 3 Input data for the uncertainty estimator.

\begin{tabular}{ccccc}
\hline Item & $\phi_{0}$ & $\phi_{1}$ & $U_{\phi}$ & $p$ \\
\hline $\min \left(\alpha_{\mathrm{C}}(\mathrm{D})\right)$ & 0.710 & 0.739 & $15.2 \%$ & 2.00
\end{tabular}

TABLE 4 Results of the uncertainty estimation.

bubble phase fraction $\alpha_{\mathrm{d}}(r)$ profiles obtained with grid-4 in Section 4.1 against the experimental measurements [55] for sampling levels D, I and M. All three figures display an overall good agreement with the experimental values. At level $\mathrm{D}$, there is a slight shift in the peak value towards the centre of the pipe, that is mainly to the initialisation procedure. As previously mentioned, the inlet boundary condition is initialised using the first available experimental 
measurements (using the A level results). However, the radial bubble phase fraction profile is only available for the total amount of bubbles, and not for individual bubble classes. Therefore, individual classes were initialised with the same radial profile, which was scaled down for every bubble class using the available bubble size distribution data. The described procedure introduces an error because different bubble classes should have a different radial profile, but the initial error diminishes as the bubbles travel upstream, which is visible from the results at levels I and $\mathrm{M}$.

The comparison of cross-sectional averaged bubble size distributions for sampling levels $D, I$ and $M$ is given in Figure 4. The dashed lines represent the experimental results, and the solid line bars denote the numerical results. The results suggest that the flow is breakup dominated, and the numerical results successfully reproduce the trends given by the measurements.
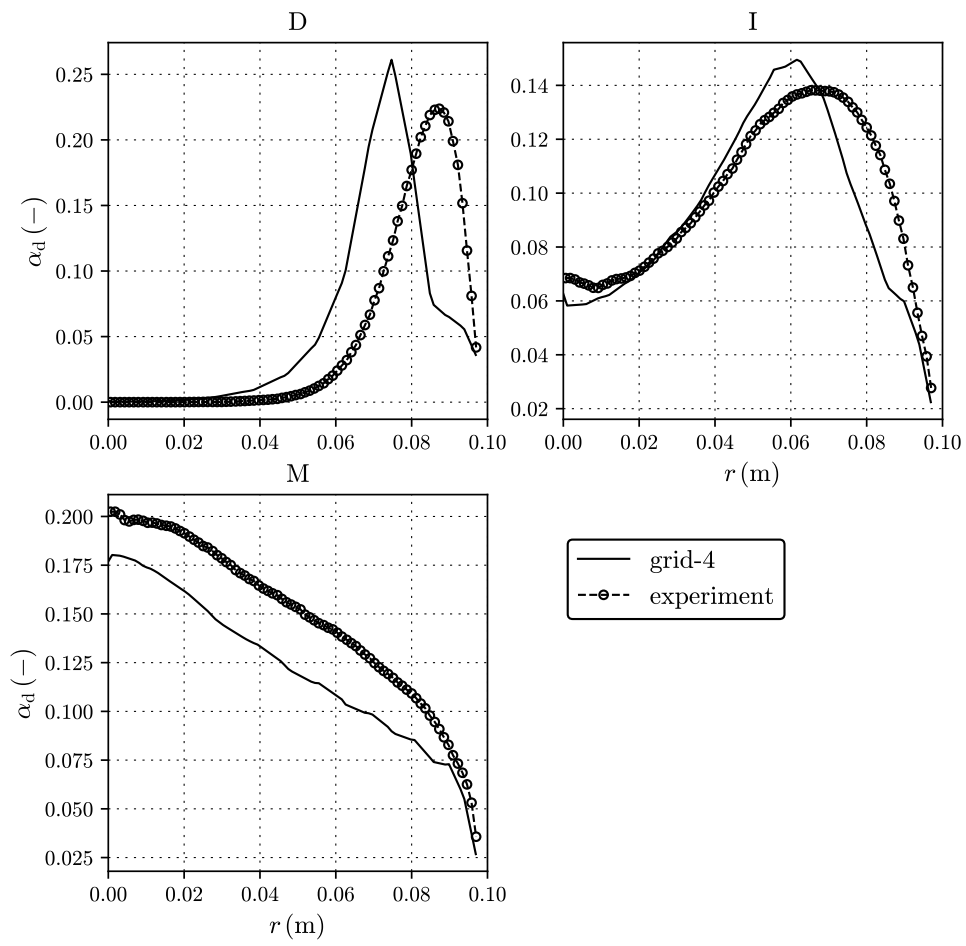

FIGURE 3 Validation: comparison of the total radial bubble phase fraction profiles.

Figure 5 shows the behaviour of the normalised residual profiles throughout the nonlinear iterations. The top plot gives the residuals of the bubble phase fractions for all of the fifteen bubble classes, and the bottom one shows the residuals for the mixture turbulence model variables and the mixture pressure.

\section{3 | Performance}

In this section, the performance of the implicitly coupled Weller formulation (CW) (eq. (19)) is compared with both segregated implementations, the segregated Weller (SW) (eq. (22)) and the standard segregated formulation (SS) (eq. (23)). To reduce the computational load of the performance test case, the number of bubble classes is reduced from 15 to 

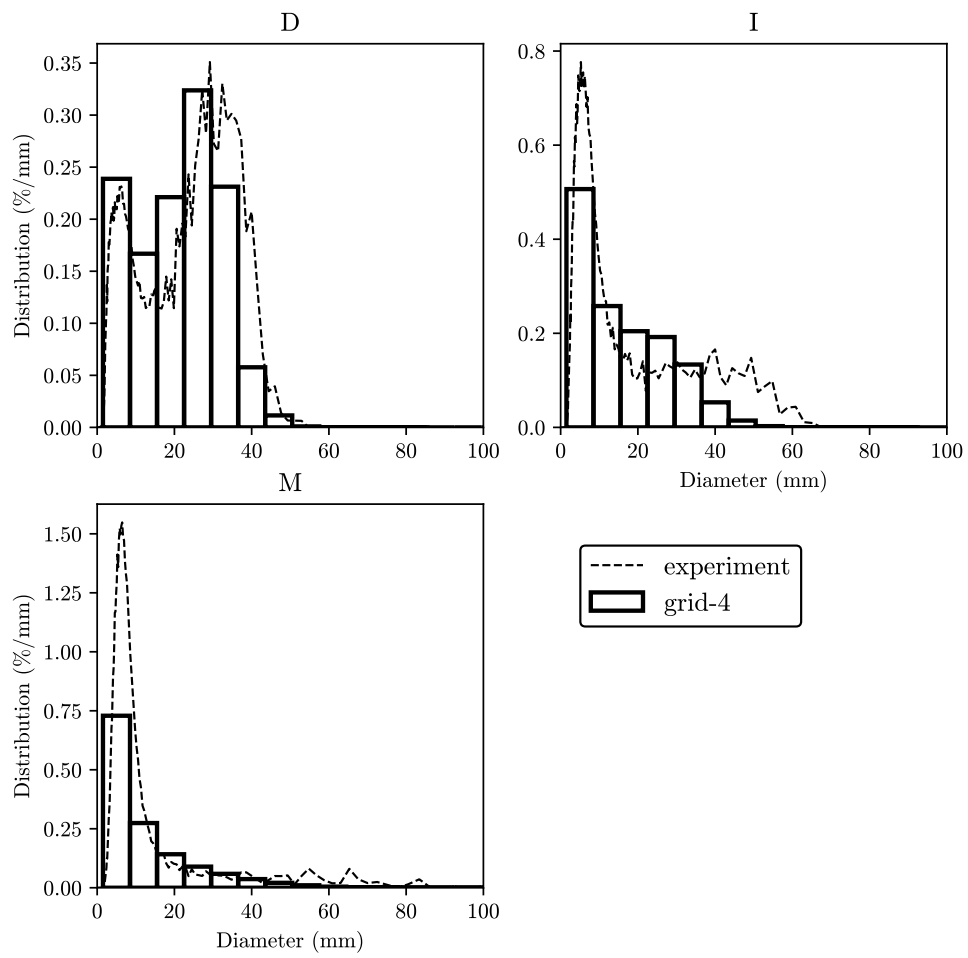

FIGURE 4 Validation: comparison of bubble size distributions.

7, and the axial length of the pipe is reduced from $5 \mathrm{~m}$ to $2 \mathrm{~m}$, but the grid is kept fine (the cell count is 6900 cells). Furthermore, to increase the cross-coupling of the phase continuity equations, the value of calibration factor $F_{\mathrm{B}}$ is increased from 0.4 to 1.

All test cases employed the same linear solver settings and same discretisation schemes (which were described in the Section 3.3), and all cases were initialised with the same initial conditions.

The performance of individual formulations is evaluated by comparing the behaviour of the relative conservation error of the continuous phase. This variable illustrates the ability of the solver to conserve the mass/volume of the continuous phase during the calculation. Due to breakup and coalescence, the mass/volume of individual bubble classes is not conserved, but the total amount of bubbles is conserved. Since $\alpha_{\mathrm{c}}$ is evaluated using the eq. (20) and eq. (21), $\alpha_{c}$ is an ideal candidate for monitoring.

Moreover, the first performance test (Section 4.3.1) also includes the comparison of the normalised residual profiles (of all variables).

The relative conservation error $e_{\mathrm{c}}$ of the continuous phase is calculated in the following manner:

$$
e_{\mathrm{C}}=\frac{\left|\alpha_{\mathrm{c}, \text { calculated }}-\alpha_{\mathrm{c}, \text { prescribed }}\right|}{\alpha_{\mathrm{c}, \text { prescribed }}}
$$

where $\alpha_{\mathrm{c}, \text { calculated }}$ is the calculated continuous phase volume fraction in the whole computational domain (volume- 

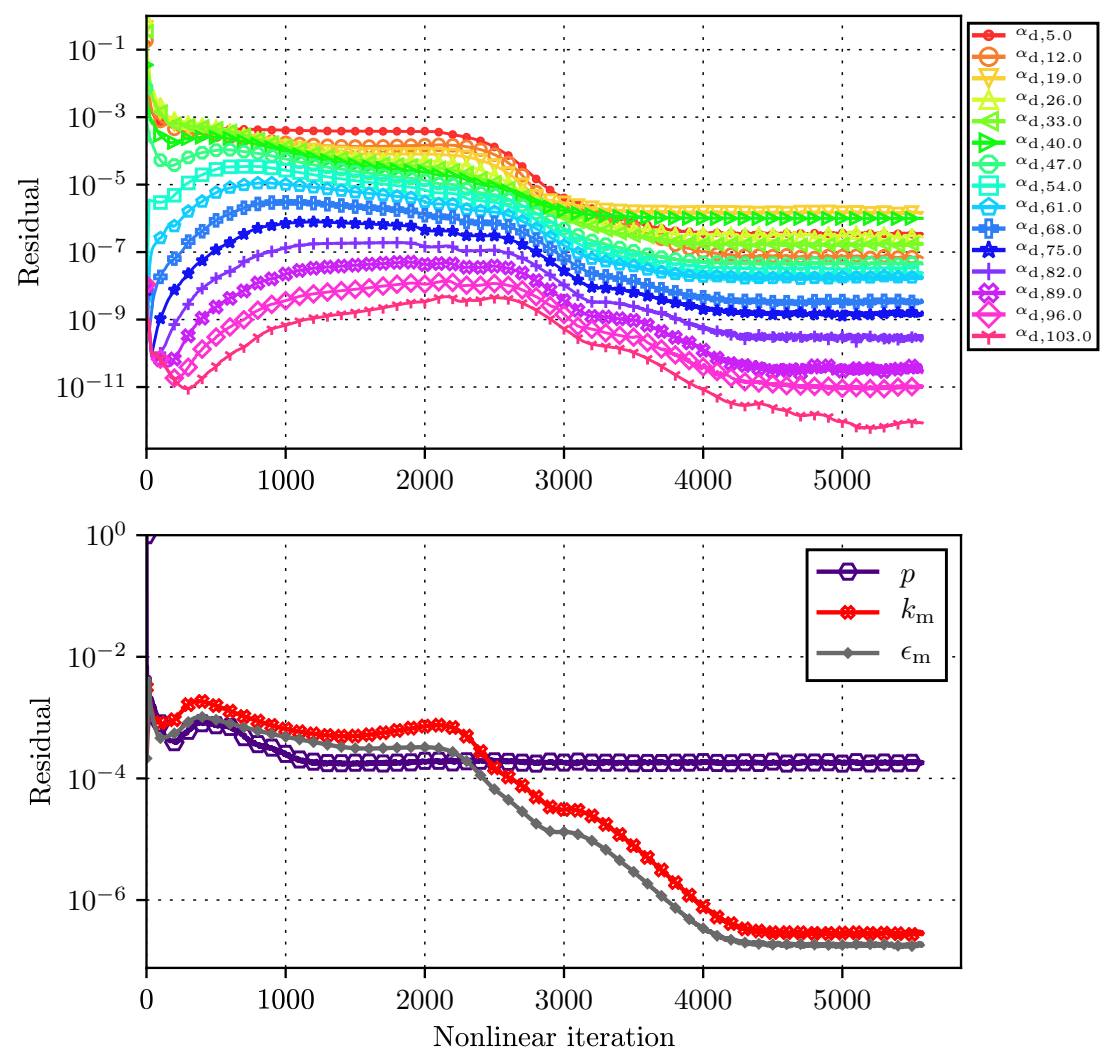

FIGURE 5 Validation: residual behaviour for grid-4.

averaged continuous phase volume fraction):

$$
\alpha_{\mathrm{c}, \text { calculated }}=\frac{\sum_{i=1}^{n_{\text {cells }}} \alpha_{\mathrm{c}}(i) V(i)}{\sum_{i=1}^{n_{\text {cells }}} V(i)}
$$

and $V(i)$ is the volume of the $i$-th cell. In eq. (24), the prescribed continuous phase volume fraction value $\alpha_{\mathrm{c}, \text { prescribed }}$ is given by

$$
\alpha_{\text {c,prescribed }}=\alpha_{\mathrm{c}, \text { prescribed }}^{\mathrm{o}}-\frac{\sum_{i=1}^{n_{\text {inlet faces }}} \alpha_{\mathrm{cf}}(i) \overline{\mathbf{U}}_{\mathrm{cf}}(i) \mathrm{S}_{\mathrm{f}}(i)+\sum_{i=1}^{n_{\text {outlet faces }}} \alpha_{\mathrm{cf}}(i) \overline{\mathbf{U}}_{\mathrm{cf}}(i) \mathrm{S}_{\mathrm{f}}(i)}{\Delta t \sum_{i=1}^{n_{\text {cells }}} V(i)},
$$

where the prescribed value from the previous time step $\alpha_{\text {c,prescribed }}^{o}$ is updated with the prescribed net increment (calculated from the continuous phase flux difference at the inlet and outlet boundaries - subscript $f$ denotes the face values of the variables). The $\Delta t$ term indicates the time step value, and the prescribed value from the previous time 
step reads:

$$
\alpha_{\mathrm{c}, \text { prescribed }}^{\mathrm{o}}= \begin{cases}\alpha_{\mathrm{c}, \text { calculated }}(t=0) & t=0 \\ \alpha_{\mathrm{c}, \text { prescribed }}(t-\Delta t) & t>0\end{cases}
$$

Furthermore, in eq. (26), the negative sign is a consequence of the OpenFOAM convention that the positive direction is the one going out of the domain.

\subsubsection{One outer corrector}

In this sub-section, the previously described test case is calculated by employing only one outer corrector, which means that the equations are solved only once per time step. The comparison of residual convergence profiles can be seen in Figure 6. The top plot presents the profiles for bubble classes, whereas the bottom one presents the profiles for the mixture pressure and mixture turbulence model variables. Solid lines represent the CW plots, dotted the SW formulation and dashed the SS formulation. It can be seen that all three formulations have practically identical convergence profiles. Therefore, it is evident that for this flow conditions, different formulations of the phase fraction equations have minimal impact on the convergence behaviour, and the convergence performance is limited with the response of the pressure-velocity coupling. In Figure 6, after approximately eight hundred nonlinear iterations there is a noticeable change in the response of residual profiles, which is directly correlated to point when the bubbles start exiting the domain, i.e. the solver is approaching a steady-state solution.

Figure 7 shows the behaviour of the relative conservation error of the continuous phase over the nonlinear iterations. Again, solid lines denote the CW formulation, dotted the SW formulation and dashed the SS formulation. The two non-implicit formulations give an almost identical error profile, whereas the implicitly coupled formulation contributed to a more conservative solution (almost two orders of magnitude lower error during a large portion of the simulation time). However, after around eight hundred iterations, i.e. when the bubble phases reach the outlet boundary, all formulations reach practically the same error level. Hence, for the described flow conditions, all formulations are limited with the conservativeness of the outlet boundary condition, but overall the coupled formulation proved to be better at providing a more conservative solution.

\subsection{2 | Two outer correctors}

This sub-section uses the same test case as Section 4.3.1, but during the calculation, it employs two outer correctors, which means that the equations are solved twice per time step. The comparison of the relative conservation error behaviour of the continuous phase over the nonlinear iterations can be seen in Figure 8. Similar to the previous test case, the implicit coupling contributes to a lower conservation error, but in this case, the difference is smaller. Repeatedly, all formulations reach the same error level once the bubble classes hit the outlet boundary condition. When comparing Figure 7 with Figure 8, it can be seen that an additional outer corrector contributes to a lower error, especially at the beginning of the calculation, and the end where the final peak value is significantly lower.

\subsection{3 | Three outer correctors}

In this sub-section, the calculation of the previously described test case is performed with three outer correctors. Figure 9 presents the relative error behaviour. In this case, the SS formulation crashes within the first one hundred 

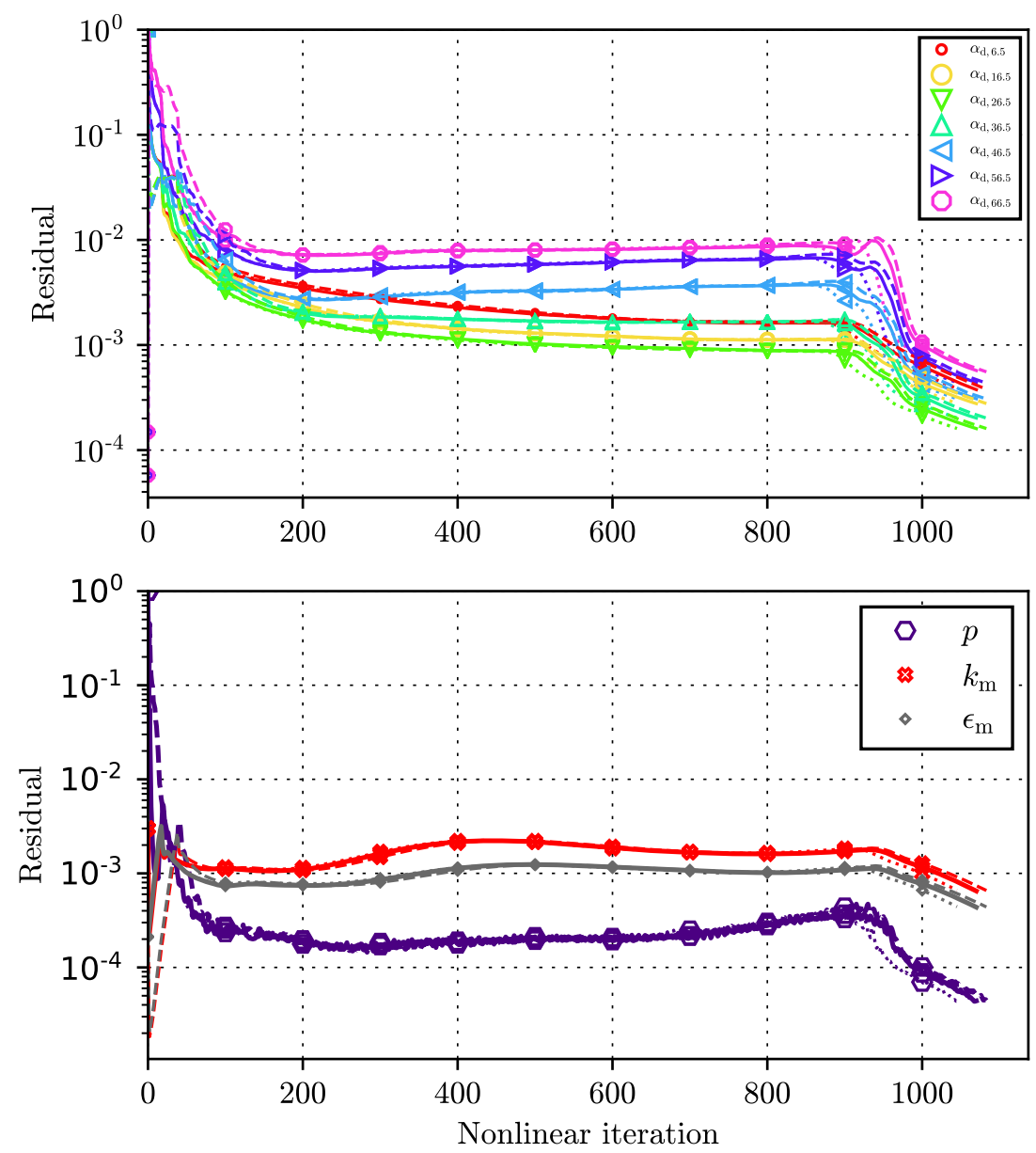

$\longrightarrow \mathrm{CW} \quad \cdots \cdots \cdot \mathrm{SW} \quad----\mathrm{SS}$

FIGURE 6 Performance: comparison of residuals convergence profiles for one outer corrector.

nonlinear iterations due to unphysical values of the bubble phase fractions. Once again, the CW formulation exhibits a more conservative behaviour until the bubbles reach the outlet boundary, after which the CW and SW formulation give very similar results.

\section{5 | CONCLUSION}

An incompressible Eulerian multi-fluid model for polydisperse flows was presented. The described model was implemented within foam-extend, a community-driven fork of OpenFOAM. The work also proposed a novel implicitly coupled phase continuity equation, which treats the breakup source and sink terms implicitly. The behaviour of the model was verified with four different computational grids for a bubbly flow with breakup and coalescence in a verti- 


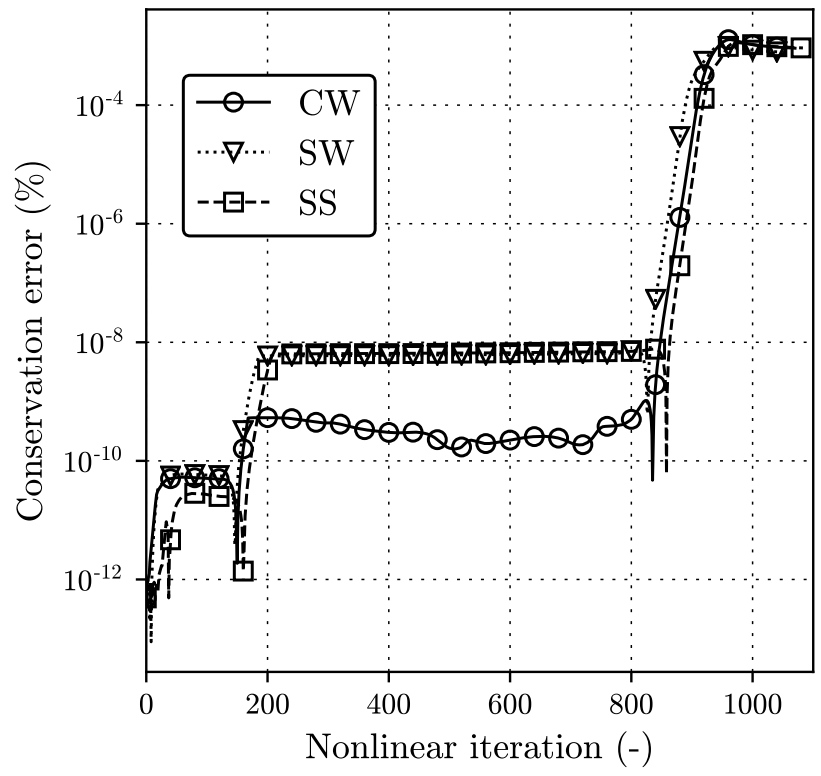

FIGURE 7 Performance: conservation error comparison for one outer corrector.

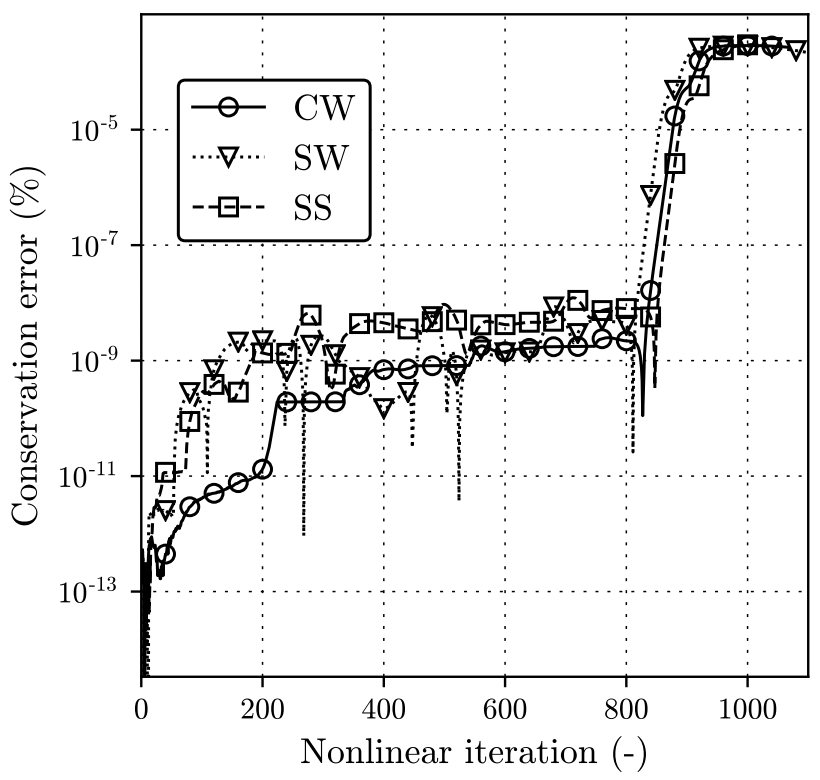

FIGURE 8 Performance: conservation error comparison for two outer corrector. 


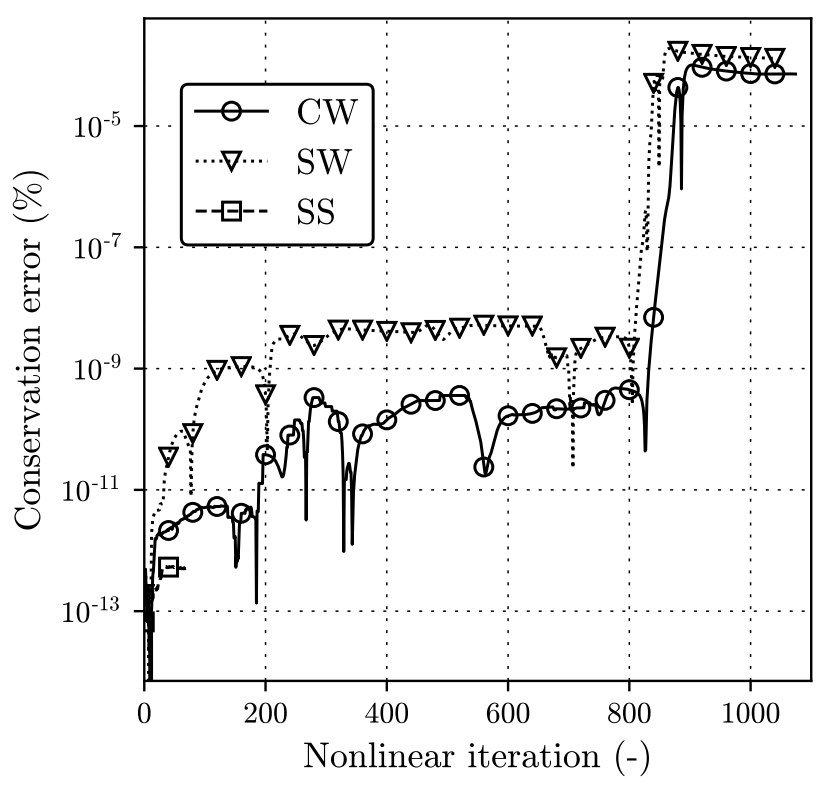

FIGURE 9 Benchmark: conservation error comparison for three outer corrector.

cal pipe. The predictive capabilities of the model proved to be stable and second-order accurate. The validation of the implemented Eulerian multi-fluid model was carried out for the flow conditions of the TOPFLOW-107 experiment. An overall good agreement was found, both for radial bubble phase profiles and the bubble size distributions. Furthermore, the performance of the novel implicitly coupled phase continuity equation was compared to the corresponding segregated formulation and the standard segregated formulation. The performance was evaluated using the relative conservation error. The implicitly coupled formulation proved to be slightly more conservative, but the performance was limited due to the behaviour at the outlet boundary conditions. Therefore, in future work, the impact of the boundary conditions will be investigated.

\section{acknowledgements}

The authors wish to thank dr. Dirk Lucas who provided valuable suggestions and comments which improved the quality of this work. This work was supported by the King Abdullah University of Science and Technology within the OSR-2017-CRG6-3409.03 research grant, and the Croatian Science Foundation (project number DOK-01-2018).

\section{references}

[1] Hirt CW, Nichols BD. Volume of fluid (VOF) method for the dynamics of free boundaries. Journal of Computational Physics 1981 jan;39(1):201-225. https://www. sciencedirect. com/science/article/pii/0021999181901455.

[2] Osher S, Sethian JA. Fronts propagating with curvature-dependent speed: Algorithms based on Hamilton-Jacobi formulations. Journal of Computational Physics 1988 nov;79(1):12-49. https://www.sciencedirect.com/science/article/ pii/0021999188900022. 
[3] Sussman M, Smereka P, Osher S. A Level Set Approach for Computing Solutions to Incompressible Two-Phase Flow. Journal of Computational Physics 1994 sep;114(1):146-159. https://www.sciencedirect.com/science/article/pii/ s0021999184711557.

[4] Han S, Zhu H. Theoretical and numerical studies on the collapse of single- and double-bubble system in water. International Journal for Numerical Methods in Fluids 2020 jul;n/a(n/a). https : //doi . org/10. 1002/fld. 4895.

[5] Subramaniam S. Lagrangian-Eulerian methods for multiphase flows. Progress in Energy and Combustion Science 2013 apr;39(2-3):215-245. https://www. sciencedirect.com/science/article/pii/s0360128512000603.

[6] Kuo TC, Pan C, Chieng CC, Yang AS. EULERIAN-LAGRANGIAN COMPUTATIONS ON PHASE DISTRIBUTION OF TWO-PHASE BUBBLY FLOWS. International Journal for Numerical Methods in Fluids 1997 mar;24(6):579-593. https: //doi.org/10.1002/(SICI) 1097-0363(19970330)24:6\{\\%\}3C579: :AID-FLD510\{\\%\}3E3.0.COhttp://2-e.

[7] Durst F, Miloievic D, Schönung B. Eulerian and Lagrangian predictions of particulate two-phase flows: a numerical study. Applied Mathematical Modelling 1984 apr;8(2):101-115. https://www.sciencedirect.com/science/article/ pii/0307904X84900623?via\{\\%\}Dihub.

[8] Hoyas S, Gil A, Margot X, Khuong-Anh D, Ravet F. Evaluation of the Eulerian-Lagrangian Spray Atomization (ELSA) model in spray simulations: 2D cases. Mathematical and Computer Modelling 2013 apr;57(7-8):1686-1693. https : //www.sciencedirect.com/science/article/pii/s0895717711006911.

[9] Subramanian K, Zalucky J, Schubert M, Lucas D, Hampel U. An Eulerian-Eulerian Computational Approach for Simulating Descending Gas-Liquid Flows in Reactors with Solid Foam Internals. Chemical Engineering \& Technology 2017 nov;40(11):2044-2057. https://doi .org/10.1002/ceat.201700136.

[10] Ziegenhein T, Rzehak R, Ma T, Lucas D. Towards a unified approach for modelling uniform and non-uniform bubbly flows. The Canadian Journal of Chemical Engineering 2017 jan;95(1):170-179. https://doi.org/10.1002/cjce.22647.

[11] Shiea M, Buffo A, Baglietto E, Lucas D, Vanni M, Marchisio D. Evaluation of Hydrodynamic Closures for Bubbly Regime CFD Simulations in Developing Pipe Flow. Chemical Engineering \& Technology 2019 aug;42(8):1618-1626. https: //doi.org/10.1002/ceat. 201900116.

[12] Crowe C, Schwarzkopf J, Sommerfeld M, Tsuji Y. Multiphase Flows with Droplets and Particles, Second Edition. CRC Press; 2011. https://www.taylorfrancis.com/books/9781439840511.

[13] Hill DP. The Computer Simulation of Dispersed Two-phase Flows. Phd thesis, Imperial College London; 1998.

[14] Meller R, Schlegel F, Lucas D. Basic verification of a numerical framework applied to a morphology adaptive multifield two-fluid model considering bubble motions. International Journal for Numerical Methods in Fluids 2020 aug;n/a(n/a). https://doi.org/10.1002/fld.4907.

[15] Tomiyama A, Tamai H, Zun I, Hosokawa S. Transverse migration of single bubbles in simple shear flows. Chemical Engineering Science 2002 jun;57(11):1849-1858. https://www.sciencedirect.com/science/article/pii/ s0009250902000854.

[16] Lucas D, Tomiyama A. On the role of the lateral lift force in poly-dispersed bubbly flows. International Journal of Multiphase Flow 2011 nov;37(9):1178-1190. https://www.sciencedirect.com/science/article/abs/pii/ s0301932211001169.

[17] Marchisio DL, Fox RO. Computational Models for Polydisperse Particulate and Multiphase Systems; 2013.

[18] Jareteg K, Sasic S, Vinai P, Demazière C. A numerical framework for bubble transport in a subcooled fluid flow. Journal of Computational Physics 2017;345:373-403.

[19] Jareteg K. Development of fine-mesh methodologies for coupled calculations in Light Water Reactors. PhD thesis, Chalmers University of Technology; 2017. 
[20] Liao Y, Oertel R, Kriebitzsch S, Schlegel F, Lucas D. A discrete population balance equation for binary breakage. International Journal for Numerical Methods in Fluids 2018 jun;87(4):202-215. https://doi .org/10.1002/fld.4491.

[21] Liao Y. Update to the MUSIG model in ANSYS CFX for reliable modelling of bubble coalescence and breakup. Applied Mathematical Modelling 2020 jan;https ://www. sciencedirect.com/science/article/abs/pii/s0307904X20300330.

[22] Heylmun JC, Kong B, Passalacqua A, Fox RO. A quadrature-based moment method for polydisperse bubbly flows. Computer Physics Communications 2019;244:187-204. http://www.sciencedirect.com/science/article/ $\mathrm{pii/S0010465519301924.}$

[23] Askari E, Proulx P, Passalacqua A. Modelling of Bubbly Flow Using CFD-PBM Solver in OpenFOAM: Study of Local Population Balance Models and Extended Quadrature Method of Moments Applications. ChemEngineering 2018 feb;2(1):8. http://www.mdpi.com/2305-7084/2/1/8.

[24] Panicker N, Passalacqua A, Fox RO. On the hyperbolicity of the two-fluid model for gas-liquid bubbly flows. Applied Mathematical Modelling 2018 may;57:432-447. https://www.sciencedirect.com/science/article/pii/ s0307904X18300234.

[25] Ransom VH, Hicks DL. Hyperbolic two-pressure models for two-phase flow. Journal of Computational Physics 1984 jan;53(1):124-151. https://www.sciencedirect.com/science/article/pii/0021999184900561.

[26] Dinh TN, Nourgaliev RR, Theofanous TG. Understanding of the III-posed two-fluid model. In: The 10th International Topical Meeting on Nuclear Reactor Thermal Hydraulics (NURETH-10) Seoul, Korea,; 2003. https://inis.iaea.org/ search/search.aspx?orig\{\_\}q=RN : 36067354.

[27] Jareteg K, Ström H, Sasic S, Demazière C. On the dynamics of instabilities in two-fluid models for bubbly flows. Chemical Engineering Science 2017 oct;170:184-194. https : //www . sciencedirect. com/science/article/pii/ s0009250917302439.

[28] Ishii M, Hibiki T. Thermo-fluid dynamics of two-phase flow; 2006.

[29] Ferreira GGS, Lage PLC, Silva LFLR, Jasak H. Implementation of an implicit pressure-velocity coupling for the Eulerian multi-fluid model. Computers and Fluids 2019 jan;181:188-207. https : //www.sciencedirect. com/science/article/ abs/pii/s0045793019300143.

[30] Weller HG. Derivation modelling and solution of the conditionally averaged two-phase flow equations. Nabla Ltd.; 2002.

[31] Oliveira PJ, Issa RI. Numerical aspects of an algorithm for the Eulerian simulation of two-phase flows. International Journal for Numerical Methods in Fluids 2003;43(10-11):1177-1198. https ://onlinelibrary. wiley.com/doi/abs/10. 1002/fld. 508 .

[32] Keser R, Vukčević V, Battistoni M, Im H, Jasak H. Implicitly coupled phase fraction equations for the Eulerian multi-fluid model. Computers \& Fluids 2019 oct;https : //doi .org/10.1016/j . compfluid.2019. 104277.

[33] Uroić T, Jasak H. Block-selective algebraic multigrid for implicitly coupled pressure-velocity system. Computers and Fluids 2018 may;167:100-110. https ://www.sciencedirect.com/science/article/abs/pii/s0045793018300847.

[34] Fernandes C, Vukčević V, Uroić T, Simoes R, Carneiro OS, Jasak H, et al. A coupled finite volume flow solver for the solution of incompressible viscoelastic flows. Journal of Non-Newtonian Fluid Mechanics 2019 mar;265:99-115. https : //www.sciencedirect.com/science/article/pii/s0377025717304937.

[35] Rusche H. Computational Fluid Dynamics of Dispersed Two-Phase Flows at High Phase Fractions. Phd thesis, Imperial College London; 2002.

[36] Lo S. Application of Population Balance to CFD Modeling of Bubbly Flow Via the MUSIG Model; 1996. 
[37] Moukalled F, Darwish M. Mixing and evaporation of liquid droplets injected into an air stream flowing at all speeds. In: Physics of Fluids, vol. 20; 2008.

[38] Duan XY, Cheung SCP, Yeoh GH, Tu JY, Krepper E, Lucas D. Gas-liquid flows in medium and large vertical pipes. Chemical Engineering Science 2011 mar;66(5):872-883. https://www.sciencedirect.com/science/article/pii/ s0009250910006937.

[39] ANSYS CFX. ANSYS CFX-Solver Theory Guide V18.2; 2017.

[40] Tomiyama A. Struggle with computational bubble dynamics. Multiphase Science and Technology 1998;.

[41] Frank T, Shi JM, Burns AD. Validation of Eulerian multiphase flow models for nuclear safety applications. In: 3rd International Symposium on Two-Phase Flow Modelling and Experimentation Pisa, 22-24 September 2004; 2004 . .

[42] Silva LFLR, Lage PLC. Development and implementation of a polydispersed multiphase flow model in OpenFOAM. Computers and Chemical Engineering $2011 \mathrm{dec}$;35(12):2653-2666. https://www.sciencedirect.com/science/article/ pii/s0098135411001451.

[43] Kissling K, Springer J, Jasak H, Schütz S, Urban K, Piesche M. A coupled pressure based solution algorithm based on the volume-of-fluid approach for two or more immiscible fluids. European Conference on Computational Fluid Dynamics 2010;(June):14-17.

[44] Luo H, Svendsen H. A Theoretical Model for Drop or Bubble Breakup in Turbulent Dispersions, vol. 42; 1996.

[45] Prince MJ, Blanch HW. Bubble coalescence and break-up in air-sparged bubble columns. AIChE Journal 1990 oct;36(10):1485-1499. https://doi.org/10.1002/aic.690361004.

[46] Behzadi A, Issa RI, Rusche H. Modelling of dispersed bubble and droplet flow at high phase fractions. Chemical Engineering Science 2004 feb;59(4):759-770. https://www.sciencedirect.com/science/article/pii/S0009250903005694? via\{\\%\}3Dihub.

[47] Jasak H. Error Analysis and Estimation for the Finite Volume Method with Applications to Fluid Flows. PhD thesis; 1996.

[48] Ferziger JH, Peric M, Leonard A. Computational Methods for Fluid Dynamics. Physics Today 1997;.

[49] Patankar SV, Spalding DB. A calculation procedure for heat, mass and momentum transfer in three-dimensional parabolic flows. International Journal of Heat and Mass Transfer 1972 oct;15(10):1787-1806. https ://www . sciencedirect . com/ science/article/abs/pii/0017931072900543.

[50] Issa RI. Solution of the implicitly discretised fluid flow equations by operator-splitting. Journal of Computational Physics 1986 jan;62(1):40-65. https://www. sciencedirect.com/science/article/pii/0021999186900999.

[51] Patankar SV. Numerical heat transfer and fluid flow. Taylor \& Francis; 1980.

[52] Keser R. Block-Coupled Solution Algorithms for 2-equation Turbulence Models. Master thesis, Faculty of Mechanical Engineering and Naval Architecture, University of Zagreb; 2016.

[53] Saad Y. Iterative Methods for Sparse Linear Systems. 2nd ed. Philadelphia, PA, USA: Society for Industrial and Applied Mathematics; 2003.

[54] Uroić T, Jasak H. Parallelisation of selective algebraic multigrid for block-pressure-velocity system in OpenFOAM. Computer Physics Communications 2021;258:107529. http://www.sciencedirect.com/science/article/ $\mathrm{pii} / \mathrm{S} 0010465520302526$.

[55] Lucas D, Beyer M, Szalinski L, Schütz P. A new database on the evolution of air-water flows along a large vertical pipe. International Journal of Thermal Sciences 2010 apr;49(4):664-674. https://www.sciencedirect.com/science/ article/pii/S1290072909002464\{\\#\}! 
[56] ReFRESCO, A community based open-usage and open-source CFD code for the Maritime World.; 2018. http://www . refresco.org/verification-validation/utilitiesvv-tools/.

[57] Eça L, Hoekstra M. A procedure for the estimation of the numerical uncertainty of CFD calculations based on grid refinement studies. Journal of Computational Physics 2014 apr;262:104-130. https : / www . sciencedirect. com/science/ article/pii/s0021999114000278. 